\title{
SEIZURES, FOCAL EEG ABNORMALITIES, AND CT
}

The prevalence and nature of abnormalities on CT associated with focal EEG abnormalities were evaluated in 964 consecutive children with epileptic and non-epileptic seizures seen over one year at the Royal Liverpool Children's Hospital, England. Of 157 (16\%) children with a focus on the EEG, 83 had slow waves and 74 had spike or sharp wave foci. Of 121 with CT head scans, $26(21 \%)$ scans were abnormal, and $81 \%$ of abnormalities were localized. Abnormal scans were uncommon in children with a single seizure, occurring in $8 \%$. Frequency of abnormal scans in patients with delta foci $(25 \%)$ was not significantly different from those with spike foci (17\%). Focal neurological signs or partial seizures were predictive of abnormal scans. Focal CT findings prompted surgery in two patients with drug resistant partial seizures but lacking focal neurological signs; one had a parietal astocytoma, the other a frontal encephalocele. (Gibbs J, Appleton RE et al. Focal electroencephalographic abnormalities and computerized tomography findings in children with seizures. I Neurol Neurosurg Psychiatry April 1993; $\underline{56}$ : 368-371). (Respond: Dr RE Appleton, Department of Paediatric Neurology, Royal Liverpool Children's Hospital, Eaton Road, Liverpool L12 2AP, England).

COMMENT. The authors advise CT in children with intractable partial seizures or lateralizing neurological findings or both, but not necessarily in all those with focal EEG abnormalities. Their findings suggest that no distinction should be made between delta and spike wave foci as criteria for CT and exclusion of neurosurgical lesion.

These results confirm a study of the electroencephalogram in 50 children with intracranial tumors and seizures reported from the Mayo Clinic (Millichap JG, Bickford RG, Miller RH, Backus RE. Neurology May 1962; 12: 329-336). A delta pattern occurred in 57\%, and spike, sharp wave, or spike-and-wave seizure discharges were recorded in $32 \%$ of children with supratentorial tumors. The EEG was of localizing value in $21(88 \%)$ of 24 patients with tumors involving the cerebral cortex, and the accuracy of EEG localization was almost equal to that of pneumography. The value of the EEG in diagnostic differentiation of a brain tumor and a scar or microgyrus was less than its localizing value.

\section{SEIZURES INDUCED BY EYE CLOSURE}

A case of a 19-year-old mentally-retarded female with an unusual form of seizures induced by eye closure is reported from the Universities of Toronto and Calgary, Canada. She developed drop attacks at age 2 years and eye fluttering seizures with altered consciousness soon afterwards. Generalized tonic-clonic seizures coincided with the introduction of valproic acid therapy at age 13 years. Clobazam controlled the grand mal, but multiple absence seizures and status persisted, despite trials with numerous antiepileptic drugs. 
The voluntary act of eye closure was a trigger for seizure activity, but visual input was an inhibitor. Unless visual input was prevented by allowing the eyes to close, seizures did not occur. Darkness alone was insufficient to elicit a seizure. The EEG seizure pattern consisted of generalized high-amplitude beta activity alternating with delta, and typical spike-and-wave activity was absent. (Barclay CL et al. Unusual form of seizures induced by eye closure. Epilepsia March/April 1993; 34: 289-293). (Reprints: Dr CL Barclay, Toronto Hospital, Western Division MP, 11th Fl, 399 Bathurst St, Toronto, Ontario, Canada M5T 2S8).

COMMENT. Seizures induced by eye closure are of two types: 1) those related to movement of the eyelids and occurring in photosensitive patients; and 2) a non-photosensitive type with scotosensitive epilepsy triggered by darkness alone or by obstruction of central visual fixation. The above patient required a combination of these two mechanisms for seizure induction.

\section{NONEPILEPTIC SEIZURES DURING SLEEP}

Nonepileptic (psychogenic or pseudo) seizures (NESs) were documented by video-electroencephalographic monitoring during apparent sleep in 12 patients reported from New York University School of Medicine. Onset was in childhood in 6 patients, and age at testing ranged from 11 to 67 years (mean, 33 yr). In addition to NESs unassociated with ictal EEG changes, 3 patients had EEG interictal epileptiform discharges and 1 had a complex partial seizure during monitoring, distinct from NES. Most NESs occurred within 2 hours of falling asleep or awakening. Provocative testing with suggestion reproduced nocturnal attacks in 9 patients. Seizures reported during sleep are not necessarily epileptic in nature. (Thacker $\mathrm{K}$ et al. Nonepileptic seizures during apparent sleep. Ann Neurol April 1993; $\underline{33:}$ 414-418). (Respond: Orrin Devinsky MD, Dept of Neurology, Hospital for Joint Diseases, 301 E 17th St, New York, NY 10003).

COMMENT. One-half of these patients had a history of childhood abuse. Four patients had evidence of epilepsy in addition to NESs.

The differential diagnosis in pseudoepileptic seizures is illustrated by case-reports from the Department of Neurology, University of Virginia Health Sciences Center, Charlottesville, VA.(Ozkara C, Dreifuss FE. Epilepsia March/April 1993; 34: 294-298). Epilepsy and pseudoepileptic seizures frequently coexist; the combination occurs in $20 \%$ before age 10 years and $60 \%$ after 10 years. Munchausen's syndrome often presents as pseudoepilepsy. Frontal lobe seizures have atypical patterns and may masquerade as pseudoseizures; these consist of thrashing, pedaling, kicking, or thrusting movements, an agitated appearance and utterance of loud expletives during sleep. 